\title{
SARS-CoV-2-associated cold agglutinin disease: a report of two cases
}

\author{
Tessa Huscenot ${ }^{1} \cdot$ Joris Galland ${ }^{1} \cdot$ Margot Ouvrat $^{2} \cdot$ Mathias Rossignol $^{2} \cdot$ Stéphane Mouly $^{1} \cdot$ Damien Sène $^{1} \cdot$ on behalf \\ of the APHP Lariboisière COVID Group
}

Received: 13 May 2020 / Accepted: 3 June 2020 / Published online: 26 June 2020

(C) Springer-Verlag GmbH Germany, part of Springer Nature 2020

\section{Dear Editor,}

Hematological manifestations reported in SARS-CoV-2 infection mainly include lymphocytopenia as a poor prognosis factor [1]. Thrombocytopenia can be associated with disseminated intravascular coagulation [2] or immune process [3]. Data regarding anemia are scarce and in line with a recent report on six patients with heterogeneous hemolytic anemia [4], we herein report on two patients with SARS-CoV-2related cold agglutinin disease (CAD).

Patient 1 A 43-year-old woman was admitted after a 10-day course of asthenia, fever, cough, diarrhea, and dyspnea. Her medical history comprised obesity and untreated multiple sclerosis. Nasopharyngeal Reverse Transcriptase-Polymerase Chain Reaction (RT-PCR) confirmed SARS-CoV-2 infection. Chest computed tomography showed a typical severe interstitial pneumonia. Hemoglobin was normal upon admission $(13.1 \mathrm{~g} / \mathrm{dL})$. A treatment with oxygen support and antibiotics (ceftriaxone and azithromycin for 3 days then tazocillin for 3 days) was started, with a rapid improvement of respiratory parameters. At day 6, hemoglobin levels dropped to $6.1 \mathrm{~g} / \mathrm{dL}$, with hemolytic features (low haptoglobin, elevated bilirubin, and lactate dehydrogenase (LDH) levels). The direct antiglobulin test (4+) was positive with the presence of cold agglutinins (Fig. 1). Etiological workup for hemolysis was negative.
After poor initial transfusion efficiency, hemoglobin levels and hemolytic parameters improved.

Patient 2 A 63-year-old man, with a medical history of hypertension, was admitted for severe acute respiratory syndrome in intensive care unit (ICU). In the last 2 weeks, he presented with fever, cough, and progressive worsening dyspnea. The nasopharyngeal RT-PCR confirmed a SARS-CoV-2 infection. The initial blood count showed a non-regenerative normocytic anemia $(10.5 \mathrm{~g} / \mathrm{dL})$. As haptoglobin levels were unmeasurable $(<0.08 \mathrm{~g} / \mathrm{L})$, contrasting with increased serum orosomucoid $(2.35 \mathrm{~g} / \mathrm{L})$, a hemolytic process was suspected and the direct antiglobulin test was positive $(\mathrm{C} 3[4+]$ and $\mathrm{IgG}$ [2+]) with cold agglutinins (Fig. 1). At day 6 of hospitalization, hemoglobin level decreased to $8.2 \mathrm{~g} / \mathrm{dL}$. Etiological workup was negative. Hemoglobin improved within 9 days along with clinical improvement.

We here report two supplemental cases of SARS-CoV-2associated autoimmune hemolytic anemia with cold agglutinins which seems to be a rare but nevertheless real complication of this condition. CAD was previously reported in association with viral infections and Mycoplasma pneumonia infection. The pathophysiological mechanism might be related to an antigen cross-reaction with red blood cell secondary to

Tessa Huscenot and Joris Galland contributed equally to this work.

Damien Sène

damin.sene@aphp.fr

1 Department of Internal Medicine, Hôpital Lariboisière, Assistance Publique Hôpitaux de Paris, University of Paris, 2 rue Ambroise Paré, 75010 Paris, France

2 Department of Intensive Care, Hôpital Lariboisière, Assistance Publique Hôpitaux de Paris, University of Paris, Paris, France 

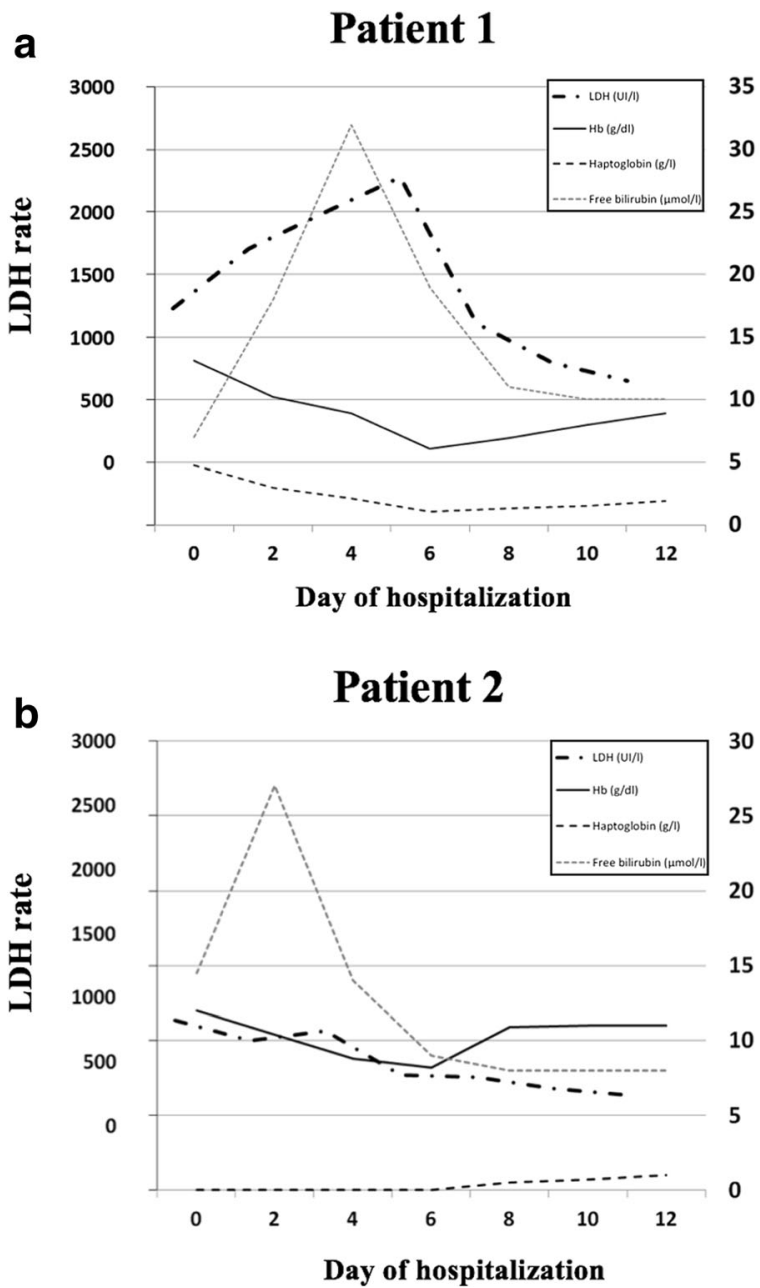

Fig. 1 Evolution of the biological parameters of cold agglutininassociated hemolysis. a Evolution of the biological parameters of hemolysis in patient $1 ; \mathbf{b}$ evolution of the biological parameters of hemolysis in patient 2

molecular mimicry, as with Influenzae disease [5]. A druginduced CAD was considered uncommon [6].

We believe that a more extensive investigation of anemia in SARS-CoV2 patients, including the appraisal of hemolytic patterns might be useful for the detection of other cases of
CAD and appropriate management of anemia during SARSCoV2-infection.

Acknowledgments The authors would like to acknowledge the patients and members of the APHP Lariboisière COVID Group (physicians, nonmedical staff).

Author contribution H.T., G.J., O.M., and S.D. designed the study, recruited patients, wrote, and reviewed, and R.M. and M.S. designed, reviewed, and approved the paper.

\section{Compliance with ethical standards}

Conflict of interest The authors declare that they have no conflict of interest.

Ethical approval All procedures performed in studies involving human participants were in accordance with the ethical standards of the institutional and/or national research committee and with the 1964 Helsinki declaration and its later amendments or comparable ethical standards.

\section{References}

1. Henry BM (2020) COVID-19, ECMO, and lymphopenia: a word of caution. Lancet Respir Med 8:e24

2. Tang N, Li D, Wang X, Sun Z (2020) Abnormal coagulation parameters are associated with poor prognosis in patients with novel coronavirus pneumonia. J Thromb Haemost 18:844-847

3. Zulfiqar AA, Lorenzo-Villalba N, Hassler P et al (2020) Immune thrombocytopenic purpura in a patient with Covid-19. N Engl J Med 382(18):e43

4. Lazarian G, Quinquenel A, Bellal M et al (2020) Autoimmune hemolytic anemia associated with Covid-19 infection. Br J Haematol. https://doi.org/10.1111/bjh.16794

5. Schoindre Y, Bollée G, Dumont M-D, Lesavre P, Servais A (2011) Cold agglutinin syndrome associated with a 2009 influenza a H1N1 infection. Am J Med 124:e1-e2

6. Swiecicki PL, Hegerova LT, Gertz MA (2013) Cold agglutinin disease. Blood 122:1114-1121

Publisher's note Springer Nature remains neutral with regard to jurisdictional claims in published maps and institutional affiliations. 